\section{Legionella pneumophila as Cause of Severe Community- Acquired Pneumonia, China}

\author{
Huahua Yi, Jie Fang, Jingwen Huang, Bing Liu, \\ Jieming Qu, Min Zhou
}

Author affiliation: Ruijin Hospital, Shanghai, China

DOI: https://doi.org/10.3201/eid2601.190655

We report a case of community-acquired pneumonia in a patient in China. We verified Legionella pneumophila infection through next-generation sequencing of blood, sputum, and pleural effusion samples. Our results show the usefulness of next-generation sequencing and of testing different samples early in the course of illness to identify this bacterium.

Community-acquired pneumonia (CAP) can lead to high mortality rates worldwide (1). Possible causes are Streptococcus pneumoniae, Haemophilus influenzae, Mycoplasma chlamydia, and Legionella spp. bacteria, as well as various respiratory viruses; although identifying pathogens in a timely manner is critical, it is not done in most cases. Next-generation sequencing (NGS) has emerged as a high-throughput method of pathogen identification and is superior to current microbiologic diagnostic measures for identifying hardto-culture pathogens. We report a case of CAP in China caused by Legionella pneumophila, determined through NGS of sputum, blood, and pleural effusion samples.

The patient was a 65-year-old woman with a history of breast cancer who sought care for fever on December 1, 2018, in a hospital in her community (Appendix 1, https://wwwnc.cdc.gov/EID/ article/26/1/19-0655-App1.pdf); her fever was accompanied by vomiting. Emergency room staff diagnosed gastroenteritis and prescribed ceftriaxone. The next day, she experienced frequent urination and urinary pain followed by diarrhea and returned to the hospital. A computed tomography (CT) scan of her abdomen showed gallbladder calculi and a lowdensity node in the right lobe of the liver. Treatment did not resolve fever and diarrhea. On the fifth day, the patient experienced respiratory symptoms including cough and purulent sputum and was admitted to the gastroenterology department in the same hospital. Over the next 2 days, respiratory symptoms worsened, and she was transferred to the infectious disease department of Ruijin Hospital in Shanghai.

At admission, PCR and serologic testing were performed as well as bacterial culture from blood and sputum samples. Chest $\mathrm{CT}$ results showed patchy consolidation in the lower lobe of the left lung (Figure, panels A and B). The patient was transferred to respiratory intensive care. A respiratory clinician, suspecting L. pneumophila as the cause, prescribed the quinolone moxifloxacin. We repeated serologic tests for L. pneumophila and cultured respiratory samples again. We sent samples of blood, sputum, and pleural effusion to BGI Group (Shenzhen, China) for NGS, and urine to Shanghai East Hospital for L. pneumophila antigen testing. Serum antibody and urine antigen tests were negative. but NGS indicated L. pneumophila in blood, sputum, and pleural effusion. For blood, the coverage rate of $L$. pneumophila was $3.2 \%$ with 1,136 raw reads; it was 3.8\% with 1,353 raw reads for sputum and $8 \%$ with 2,867 raw reads for pleural effusion (Appendix 2 Tables 1-3, https://wwwnc.cdc.gov/EID/article/26/1/19-0655App2.xlsx). Under moxifloxacin treatment, the patient's symptoms disappeared in 1 week. We conducted another NGS for blood without any evidence for L. pneumophila. Patchy consolidation in the left lower lung lobe was almost completely absorbed (Figure 1, panel C).

We confirmed the presence of L. pneumophila bacteria by PCR for the remaining pleural infusion and sputum of this patient. We did not investigate the source of the L. pneumophila bacteria.

L. pneumophila bacteria is 1 of the 3 most common pathogens that cause severe CAP and is isolated in $1 \%-40 \%$ of hospital-acquired pneumonia cases (2). Extrapulmonary symptoms are always present in L. pneumophila infection, which may increase the risk for misdiagnosis early in the infection. Furthermore, culturing $L$. pneumophila requires a specific medium, which makes it difficult to obtain a diagnosis without positive microbiology test results. L. pneumophila infections have been reported previously $(3,4)$ but were confirmed by PCR with target microorganisms and not by NGS.

BGI Group conducted NGS as described previously (5), collecting 3-4 mL blood in ethylenediaminetetraacetic acid tubes, staining in RT for 3-5 minutes, and centrifuging at $1,600 \times g$ for $10 \mathrm{~min}$ at $4^{\circ} \mathrm{C}$. They then collected $0.5-3 \mathrm{~mL}$ sputum or pleural effusion sample following standard procedures and, using a $1.5-\mathrm{mL}$ microcentrifuge tube, agitated the sample at 2,800-3,200 rpm for $30 \mathrm{~min}$. They separated $0.3 \mathrm{~mL}$ of the sample into a new tube and extracted DNA using the TIANamp Micro DNA Kit (DP316; Tiangen Biotech, http://tiangen.com) according to the manufacturer's recommendation. DNA libraries were constructed through DNA fragmentation, end repair, adapter ligation, and PCR amplification.

BGI Group sequenced the qualified libraries by using the BGISEQ-100 platform (6). They generated 


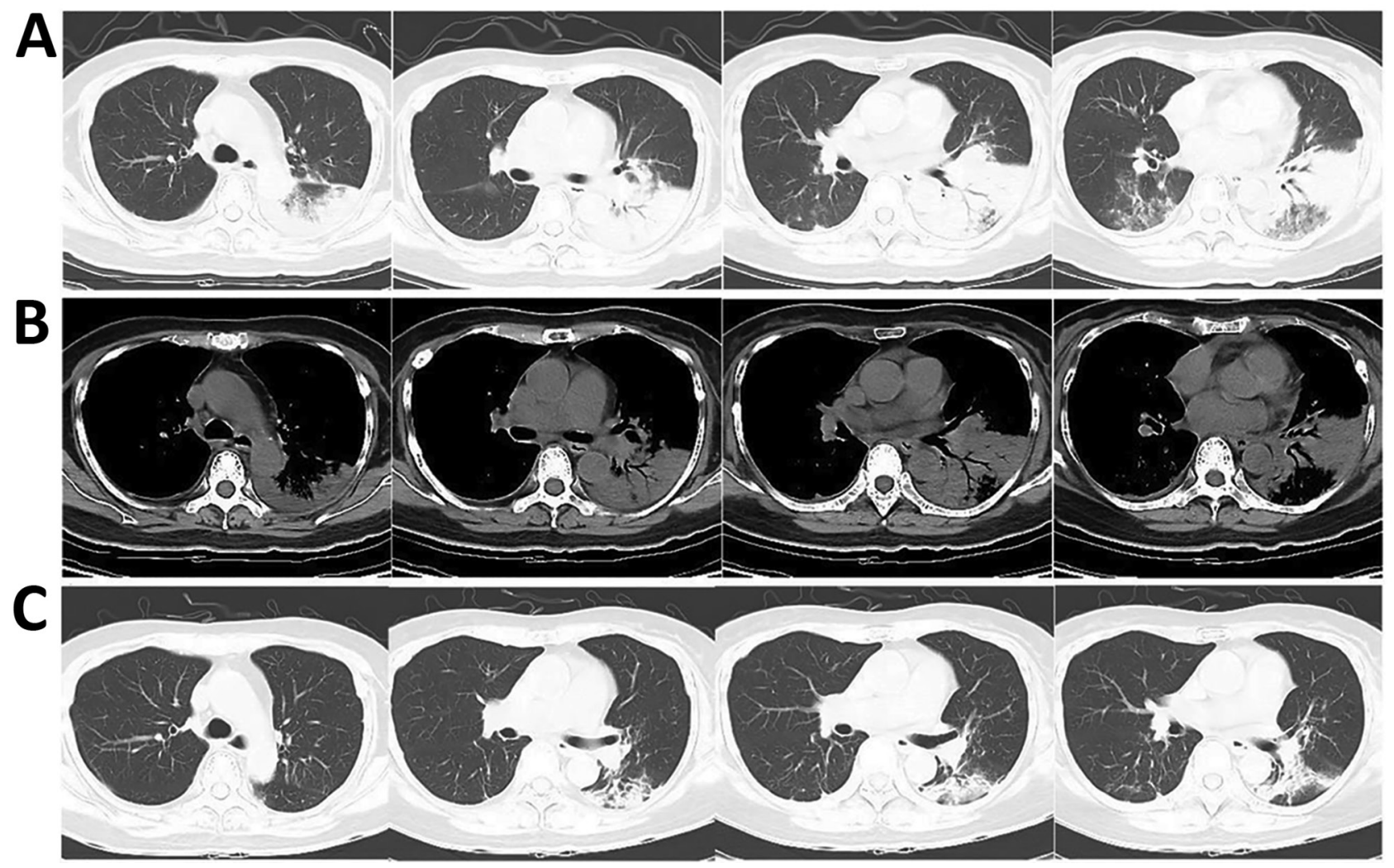

Figure. Computed tomography images of the lungs of a 65-year-old woman with community-acquired pneumonia caused by Legionella pneumophila bacteria, China, showing absorption of infusion in left lobes after effective treatment. Lung window (A) and bone window (B) at the beginning of treatment showed consolidation in the left lobes; after 1 week of treatment (C), infusion is mostly absorbed in the left lobes.

high-quality sequencing data by removing low-quality and short (length $<35 \mathrm{bp}$ ) reads, then performed computational subtraction of human host sequences mapped to the human reference genome (hg19) using Burrows-Wheeler alignment (7). They classified the remaining data by removing low-complexity reads and simultaneously aligning the sequences to microbial genome databases for bacteria, viruses, fungi, and parasites downloaded from the US National Center for Biotechnology Information (ftp:/ / ftp.ncbi. nlm.nih.gov/genomes). Experts in respiratory illness, microbiology, and radiology evaluated patients' status and interpreted results of NGS testing together, thus identifying possible etiologic agents.

Because turnaround time is short and nonspecific primers can be used, NGS is useful for detecting unculturable pathogens, especially in severely ill patients (8). NGS is highly sensitive and expensive, so clinicians must assess the value of NGS for identifying etiologic pathogens. In this case, we diagnosed L. pneumophila infection on the basis of 3 kinds of samples that had not been reported previously, which shows the importance of testing multiple samples early in the course of illness to identify the etiologic agent and begin appropriate treatment.

\section{Acknowledgments}

We thank BGI-Shenzhen for conducting next-generation sequencing and the clinicians of Shanghai East Hospital for urine antigen.

The study was supported by National Key R\&D Program of China (2017YFC1309700 and 2017YFC1309701); National Natural Science Foundation of China (81570029); Shanghai Key Discipline for Respiratory Diseases (2017ZZ02014); and the Innovative Research team of high-level local universities in Shanghai.

\section{About the Author}

Dr. Yi is a PhD candidate at Shanghai Jiao Tong University School of Medicine under the supervision of Dr. Min Zhou, specializing in respiratory infections and chronic obstructive pulmonary diseases. Her primary research interest is the diagnosis and treatment of respiratory infectious diseases, especially among immunocompromised patients. 


\section{References}

1. Prina E, Ranzani OT, Torres A. Community-acquired pneumonia. Lancet. 2015;386:1097-108. https:/ / doi.org/ 10.1016/S0140-6736(15)60733-4

2. Diederen BM. Legionella spp. and Legionnaires' disease. J Infect. 2008;56:1-12. https:/ / doi.org/10.1016/j.jinf.2007.09.010

3. Zhang Q, Zhou H, Chen R, Qin T, Ren H, Liu B, et al. Legionnaires' disease caused by Legionella pneumophila serogroups 5 and 10, China. Emerg Infect Dis. 2014;20: 1242-3. https:/ / doi.org/10.3201/eid2007.131343

4. Buchholz U, Reber F, Lehfeld AS, Brodhun B, Haas W, Schaefer B, et al. Probable reinfection with Legionella pneumophila - a case report. Int J Hyg Environ Health. 2019;222:315-8. https:// doi.org/10.1016/j.ijheh.2018.11.001

5. Miao Q, Ma Y, Wang Q, Pan J, Zhang Y, Jin W, et al. Microbiological diagnostic performance of metagenomic next-generation sequencing when applied to clinical practice. Clin Infect Dis. 2018;67:S231-S240.

6. Jeon YJ, Zhou Y, Li Y, Guo Q, Chen J, Quan S, et al. The feasibility study of non-invasive fetal trisomy 18 and 21 detection with semiconductor sequencing platform. PLoS One. 2014;9:e110240. https:/ / doi.org/10.1371/journal. pone. 0110240

7. Freitas TAK, Li P-E, Scholz MB, Chain PSG. Accurate readbased metagenome characterization using a hierarchical suite of unique signatures. Nucleic Acids Res. 2015;43:e69.

8. Naccache SN, Federman S, Veeraraghavan N, Zaharia M, Lee D, Samayoa E, et al. A cloud-compatible bioinformatics pipeline for ultrarapid pathogen identification from nextgeneration sequencing of clinical samples. Genome Res. 2014;24:1180-92. https://doi.org/10.1101/gr.171934.113

Address for correspondence: Min Zhou, Ruijin Hospital, Shanghai Jiao Tong University Medical School Affiliated, Department of Respiratory and Critical Care Medicine, No. 197 Ruijin Er Rd, Shanghai, 200025, China; email: doctor_zhou_99@163.com

\section{Training for Foodborne Outbreak Investigations by Using Structured Learning Experience}

\author{
Florian Burckhardt, Esther Kissling \\ Author affiliations: European Programme for Intervention \\ Epidemiology Training Alumni Network, Heidelberg, Germany \\ (F. Burckhardt); Epiconcept, Paris, France (E. Kissling)
}

DOI: https://doi.org/10.3201/eid2601.190755
We created a free and interactive training activity based on playing cards (disease detective cards) that introduces foodborne outbreak investigations to public health professionals and students. Competencies taught coverselected descriptive and analytic epidemiologic topics, such as case definition, epidemic curve, 2-by-2 tables, relative risks, attack rates, stratification, and confounding.

Training programs, such as the Epidemic Intelli1 gence Service of the Centers for Disease Control and Prevention (Atlanta, GA, USA) (1) and the European Programme for Intervention Epidemiology Training of the European Centre for Disease Prevention and Control (Stockholm, Sweden) (2), teach future epidemiologists outbreak investigation skills. Their case studies for foodborne outbreaks are often based on historic investigations whose real-world complexity can be overwhelming for beginners.

An introductory study (3) used 14 exposures to explain the concept of risk factors and their association with disease. We designed a scripted foodborne outbreak investigation as a structured learning experience with illustrated playing cards that depict exposures (food eaten) and outcome (symptoms, time to first symptoms). With the cards and a flipchart as props, facilitators teach core skills and competencies of outbreak investigations in an interactive group exercise.

We modeled a hypothetical Salmonella species outbreak investigation with a cohort design in Excel 2010 (Microsoft, https://www.microsoft.com). We took symptoms and incubation periods from our own unpublished outbreak investigations and literature research $(4,5)$. We reverse-engineered relative risks for univariable and stratified analysis with their 2-by2 tables into a line list with the desired properties and transcribed onto playing cards in which each card represents 1 person attending the exposure event.

Dynamic illustrations of gastroenteric symptoms and food items were hand drawn with pen and ink on Bristol board, scanned, and colored by using the GNU Image Manipulation Program (https://www. gimp.org). The illustrations depict characters from our earlier work, the disease detectives (6). We used OpenOffice software (https://www.openoffice. org) for layout of the cards. The teaching script is a restructured and simplified adaptation of outbreak case studies used during European Programme for Intervention Epidemiology Training introductory courses $(2,3)$. The cards and other resources are available (https://www.disease-detectives.org) under a Creative Commons open-culture license in multiple formats for home printing or as templates for highquality printing services. 